Published in final edited form as:

Pharmacogenomics. 2005 December ; 6(8): 857-864.

\title{
Pharmacogenomics and Drug Development
}

\author{
Yingying Guo ${ }^{1}$, Steven Shafer ${ }^{3}$, Paul Weller $^{2}$, Jonathan Usuka ${ }^{1}$, and Gary Peltz ${ }^{1}$ \\ 1Departments of Genetics and Genomics, Roche Palo Alto, Palo Alto, CA 94304
}

2Drug Metabolism and Pharmacokinetics, Roche Palo Alto, Palo Alto, CA 94304

3Department of Anesthesia, Stanford University, Stanford, CA 94304.

\begin{abstract}
It is generally anticipated that pharmacogenomic information will have a large impact on drug development and will facilitate individualized drug treatment. However, there has been relatively little quantitative modeling to assess how pharmacogenomic information could best be utilized in clinical practice. Using a quantitative model, we demonstrate that efficacy is increased and toxicity is reduced when a genetically-guided dose adjustment strategy is utilized in a clinical trial. However, there is limited information available about the genetic variables affecting the disposition or mechanism of action of most commonly used medications. These genetic factors must be identified to enable pharmacogenomic testing to be routinely used in the clinic. A recently described murine haplotype-based computational genetic analysis method provides one strategy for identifying genetic factors regulating the pharmacokinetics and pharmacodynamics of commonly used medications.
\end{abstract}

\section{INTRODUCTION}

There is widespread anticipation that pharmacogenomic information will have a large impact on drug development, and subsequently on clinical practice. Use of pharmacogenetic information may also enable medication and dosage selection to be individualized, which will improve the efficacy and reduce the toxicity of drug treatment. Despite this expectation, there has been relatively little testing and quantitative modeling of the impact of that pharmacogenomic information can have on drug development and clinical practice. There are few guideposts providing information about the type of pharmacogenomic information that should be generated, and where this information can be utilized in clinical practice. However, we can monitor progress along the 'road' to optimal utilization of pharmacogenomic information in clinical practice through a simulation that assesses what would be possible if pharmacogenomic information were available. We use a quantitative model to examine how pharmacogenomic information could impact clinical trials.

This modeling exercise demonstrates that utilization of pharmacogenomic information can significantly improve clinical drug development. However, there is limited available information on the genetic factors regulating the mechanism of action or disposition for many commonly used medications. This lack of knowledge represents a major bump on the road towards clinical utilization of pharmacogenomic information. These genetic factors must be identified and characterized to enable the routine use of pharmacogenomic testing in clinical practice. A recently described murine haplotype-based computational genetic analysis method provides one strategy for identifying genetic variables affecting the pharmacokinetics or pharmacodynamics of common drugs.

Correspondence to: Gary Peltz.

Correspondence to: Gary Peltz, M.D., Ph.D. Email: gary.peltz@roche.com Roche Palo Alto S3-1, 3431 Hillview Ave, Palo Alto, CA 94304. 
Modeling the Impact of Genetically-Guided Dose Adjustment. We illustrate the potential impact of pharmacogenomic information using a quantitative model by comparing clinical trials that are performed with and without knowledge of patient genotypes. For this model, it is assumed that a single gene with polymorphic alleles in the treated population controls a fundamental aspect of the metabolism of a hypothetical test drug. Therefore, individuals with different allelic forms of this gene have different pharmacokinetic profiles. This is similar to the clinical situation where individuals have rapid, slow, and intermediate rates of metabolism of debrisoquine, a prototypical drug whose rate of disposition is determined by genetic differences in the treated population [5].

For the simulation model, the major (+) and the minor (-) alleles for this gene have frequencies of 0.8 and 0.2 , respectively, in the treated population. Since these alleles are assumed to be in Hardy-Weinberg equilibrium, three distinct genotypes $(+/+,+/-$ and $-/-)$ of this gene make up the $64 \%, 32 \%$ and $4 \%$ of the treated population, respectively. The homozygous $(+/+)$ wildtype patients metabolize and excrete the drug rapidly, and are defined as having a normal rate of metabolism of this drug. The homozygous (-/-) individuals have a reduced rate of drug metabolism and elimination, and are defined as slow metabolizers. The (+/-) heterozygotes have an intermediate rate of drug metabolism that is between the two homozygous extremes. A simple pharmacokinetic model is applied in this simulation. Therefore, peak concentration following a bolus dose of 1 unit of drug is 1 and 2 unit/liter in the (+/+) wildtype and (-/-) homozygotes, respectively. Similarly, we assume that for the (+/-) heterozygous patient, the peak concentration after a bolus dose of 1 unit is 1.5 units/liter, reflecting equal contributions of the + and - alleles to the observed phenotypic response. Of course, there is some variability in the plasma drug concentration among individuals with the same genotype. For this simulation, we assume that the extent of variation in plasma drug concentration among individuals with the same genotype has a log normal distribution of $20 \%$ coefficient of variation (CV) in the treated population. The $20 \% \mathrm{CV}$ is an estimate that represents the mid-point between drugs (such as inhaled anesthetics) with a CV of less than 10\%, and others with a $30 \%$ $\mathrm{CV}$ in the treated population.

The recommended drug dose should produce therapeutic concentrations in as large a fraction of the treated population as possible. However, the dose that yields a therapeutic effect in the $64 \%$ of patients with the $(+/+)$ homozygous genotype might produce toxicity due to high plasma levels in the (+/-) heterozygous patients with an intermediate rate of metabolism of this drug. There is an even greater risk of toxic concentrations occurring in the (-/-) homozygous individuals with slow rate of drug metabolism. The influence of allele frequencies on the overall distribution of drug response in the treated population is illustrated in Figure 1. The (-/-) homozygous patients are at greater risk of toxicity following any given dose, since they dominate the upper part of the expected concentration range for a dose of 1 unit (Figure 1). Of course, the size of the therapeutic index and the magnitude of the toxicity under consideration determine whether or not these concentrations produce a clinically apparent problem.

How can a safe and effective dose be identified? This question can be answered through clinical trial simulation. We first need to define a minimum fraction of the population that must benefit from drug treatment for this drug to be considered efficacious, and a maximum allowable fraction of the population that can experience toxicity for the drug to be considered safe. Of course, the acceptable incidence of toxicity cannot be set at zero, because the log-normal distribution assures that some fraction of treated patients will have a drug concentration that is above any toxic threshold. However, for any toxicity, there is some threshold incidence below which the drug is considered safe, and above which the drug is considered unacceptably toxic. The minimum and maximum fractions are clinically determined, based upon the treatment benefits and the risks associated with drug toxicity. 
With the above as background, we set the following criteria for the clinical trial simulation, which will also apply to the routine use of the drug in a clinical population:

1. The lower bound of therapeutic window-the concentration below which the drug is not efficacious-is arbitrarily set at 7 units/liter

2. The lower bound on the fraction of the treated population that must experience benefit for the drug to be considered efficacious is $50 \%$

3. The upper bound of the therapeutic window-the concentration above which patients will experience toxicity-is arbitrarily set at 12 units/liter

4. The upper bound on the fraction of patients that can experience toxicity before the drug is considered unsafe: $2 \%$

To reflect the procedures that normally occur during drug development, this simulation will be performed as a sequence of two simulated trials, whose overall process and purposes are described in Figure 2. The first trial simulated will be a phase II pharmacokinetic trial whose goal is to determine the relationship between the administered dose and plasma drug concentration. Each treated subject will receive a dose of 1 unit, and then the plasma drug concentration will be measured. The intent is to identify the dose that has less than a $5 \%$ chance of producing a failed phase III trial. By definition, a failed phase III trial is one where at least $2 \%$ of the subjects experience toxicity. The dose from the phase II trial that will be used in the phase III trial is calculated in the following way. The concentration at which $98 \%$ of population does not experience toxicity is identified for each of the 250 simulations. The mean, standard deviation, and confidence bounds are then calculated using this concentration. The confidence bound is based on the number of subjects in the simulation (e.g., 10, 100, 200, 1000), and is not based on the number of simulations performed. To produce a $5 \%$ chance of a failed trial, the upper $5 \%$ of this confidence bound was divided into 12, the upper limit of an acceptable drug concentration. It was projected that this dose would produce a 5\% risk of a failed phase III pivotal trial.

Then, the pivotal phase III trial used the dose established in the phase II trial to test the efficacy and safety of the drug. For this simulation, the drug is assumed to have efficacy in the fraction of the population with a plasma drug concentration that is greater than 7 units per liter. The drug will be safe in the fraction of the population with a plasma drug concentration below 12 units per liter.

Our clinical trial simulations compared the outcome of a genetically uninformed design to a genetically informed design. These two types of clinical trial designs represent two fundamentally different strategies for clinical testing. In the genetically uninformed design, the genotype was ignored when calculating the dose for the phase III trial. In the genetically informed design, the genotype of each individual was used to adjust the dose of drug administered in the phase III trial. The simulations were performed in Excel spreadsheets (available from steven.shafer@stanford.edu), and each clinical trial was simulated 250 times. The response of each patient was calculated based upon genotype, and patient genotypes were assumed to have a Hardy-Weinberg genotype distribution as described above. The simulations were conducted for studies with a varying numbers of subjects to determine whether genetic data would change the number of subjects required to demonstrate efficacy and safety. The principles used in the phase II and III clinical trial simulations are described in text boxes I and II.

In the genetically uninformed design, the dose was modestly increased as the number of subjects was increased in the phase II simulation (table I). This reflects the smaller confidence bounds that are associated with a study that has a larger number of patients. All drug doses used in the subsequent pivotal phase III trial simulations were performed with 200 or 1000 
subjects. Overall, the efficacy of the proposed drug was quite low in all of the subsequent phase III trial simulations that did not use genetic information to adjust the dose. Fewer than $30 \%$ of drug treated subjects in a simulated clinical trial that did not use pharmacogenomic information benefited from the treatment. The incidence of toxicity and the percent of failed trials were generally within the boundaries established for the simulations. The only exception was the simulation of the highest dose in a phase III trial of just 200 subjects (Table I, last line), which resulted in both excessive toxicity (12\% of the population) and an excessive number of failed trials $(17 \%)$. This excessive toxicity was not observed in the phase III simulation of 1000 individuals. It demonstrates the risks of clinical trial simulation when the dose constructed from a well characterized confidence bound (1000 subjects in phase II simulation) is taken forward in a simulation using fewer subjects, where there is more chance for random events to alter the trial outcome.

The results of the genetically informed trial simulation are presented in table II. Because of the small number of homozygous (-/-) individuals in the treated population, at least 200 subjects must be studied in a phase II trial to guarantee that at least $1(-/)$ genotype individual is present in each of the 250 simulations. Because the genotype of each individual was measured, the phase II trial recommended a different dose for individuals with each of the three different genotypes. In every simulated trial using pharmacogenomic information to adjust the dose, more than $60 \%$ of treated patients benefited from the treatment and less than $2 \%$ experienced toxicity. These results are indicative of a successful and safe drug therapy. However, the dose calculated from the phase II trial with 1000 subjects produced an unacceptably high incidence of failed trials in the subsequent phase III trial with only 200 subjects. This is the same result that was seen in the genetically uninformed trial simulation. Therefore, even when genetic information was utilized for dose adjustment, a successful phase III trial required at least 1000 subjects to demonstrate that this drug was safe, and that there was an acceptably low risk of a failed trial.

These simulations demonstrate that incorporation of a genetically-guided dose adjustment strategy into the design of a clinical trial can significantly increase drug efficacy and reduce toxicity. Pharmacogenomic information can have an even greater impact on outcome when the drug induces a very serious adverse event. In this simulation, which characterized the relatively simple case where a two allele, single gene model of pharmacogenetic variability alters the rate of drug metabolism, utilization of pharmacogenetic information for dosage adjustment enormously altered the outcome of a clinical trial. This relatively simple simulation was presented to ensure clarity. However, utilization of pharmacogenetic information can improve clinical trial design in a wide variety of clinical scenarios, including those that are more likely to occur in a drug-treated population. For example, the same modeling process was used to analyze more genetically complex situations where two or three different alleles independently contributed to the pharmacokinetic response, and genetically-based dosage adjustment also improved clinical trial outcome (data not shown). If the genetic variant affected a pharmacodynamic response (efficacy or toxicity), a similar improvement in clinical trial outcome would result from stratification of individuals based upon this genetic variant. Furthermore, the same simulations performed using a range of different allele frequencies (data not shown) for the causative genetic variant demonstrated that pharmacogenomic information improved clinical trial outcome. This simulation assumes that the phenotype is fully determined by the genetic variant. If an allele has a low penetrance, then the utility of genetic analysis will decline.

Filling in the Potholes on the Pharmacogenomic Road. The above simulation demonstrated that treatment outcome was significantly improved through utilization of pharmacogenomic data to individualize drug dosage. An effect of similar magnitude would also be seen if the simulation was performed using a genetic variable that affected a pharmacodynamic response. 
However, our current lack of knowledge about the genetic variables that affect medication and dosage selection is a major remaining barrier to overcome on the road to pharmacogenomicdata driven clinical decision making. Therefore, it is essential that efficient strategies are developed and employed to identify genetic factors that regulate drug disposition, mechanism of action, and toxicity for many of our current and future therapies.

At first thought, it may appear that we have not advanced very far along the road to optimal utilization of pharmacogenomic information in clinical practice. In most clinical situations, medications and dosage are currently selected without the aid of pharmacogenomic data. Nevertheless, there are already some areas, such as in the treatment of breast cancer and AIDS, where pharmacogenomic measurements are now routinely used to guide drug selection. A diagnostic test measuring the level of HER 2 protein expression, identifies breast cancer patients who are more likely to respond to treatment with an antibody (trastuzumab) against this receptor $[3,17]$. Similarly, the HIV titer is now routinely measured in AIDS patients to assess the response to drug therapy. In the near future, HIV viral genotyping will routinely be used to select the optimal drug therapy for AIDS patients $[11,13]$, and patients may also be genotyped to optimize selection of HIV therapy [14]. These examples provide a few instances where pharmacogenomic data has been used to optimize treatment. However, there are many additional opportunities where inter-individual differences in drug dose or selection can be predicted by genotyping. For example, certain HLA alleles are associated with development of a life-threatening, hypersensitivity reaction that occurs in approximately 5\% of HIV patients treated with Abacavir [4]. A recently available microarray analyzes genetic variation in two genes (CYP2D6 and CYP2C19) that play a role in the metabolism of almost $25 \%$ of all prescription drugs $[5,8]$. Genotyping with this microarray may enable pharmacogenomic data to be generated that will allow individualized dosage adjustment for a large number of therapeutics. Although in its infancy, pharmacogenomic testing was incorporated in the design of a clinical trial. Genotyping of CYP2D6 alleles was performed to exclude individuals who would have difficulty metabolizing the drug from enrolling in a phase III clinical trial [12]. Although there are regulatory, financial and other barriers to overcome before pharmacogenomic testing is more widely used in clinical practice; the technical infrastructure to reproducibly analyze DNA, RNA or protein in clinical samples has been established. However, a major remaining barrier to overcome on the pharmacogenomic road is identification of genetic variables that affect the metabolism and response to many commonly used drugs.

Many drugs that are commonly used today were discovered without knowing how they work, or their molecular target. It is also not uncommon in clinical practice for the dosage of a drug to be adjusted empirically based upon the presence or absence of a therapeutic response or a toxic effect. However, several new tools have recently emerged that can increase our understanding of the factors regulating the metabolism and mechanism of action of many commonly used prescription medications. These tools should be utilized to increase the rate at which pharmacogenomic factors that could improve clinical response are discovered. As one example, libraries of yeast with haploid gene deletions have been utilized to identify the mechanism of action for several therapeutic agents $[1,2,10]$. Drug-induced phenotypic changes were used as the endpoint to identify the drug target. With this tool, 5-fluorouracil was shown to act on the rRNA processing machinery; and sphingolipid synthesis was affected by dihydromotuporamine $\mathrm{C}$, a compound in preclinical development that inhibits metastasis $[1$, $10]$.

Mouse genetics provides another efficient discovery tool that could be used to identify genetic factors regulating drug metabolism and response. Analysis of experimental murine models has provided support for many therapies used in clinical practice. We have analyzed mouse genetic models of asthma [6], systemic lupus [16] and osteoporosis [7] and identified a genetic factor 
responsible for strain-specific differences in each disease-related trait (reviewed in [15]). Inbred mouse strains also vary in their response to drug treatment [20]. All of the variables that confound pharmacogenetic analysis in human populations may be controlled in an experimental mouse model (see review [19]), and the analysis of a drug-treated mouse cohort can be completed at a fraction of the cost required for a clinical population. Despite its potential, the methods currently used for mouse genetic analysis require the costly and time consuming process of generating, phenotyping, and genotyping 300 to 1,000 experimental intercross progeny $[15,18]$. Consequently, identifying a causative genetic variant in a murine genetic model often requires a minimum of 5 years, and usually involves at least 5 scientists.

To reduce the cost and time requirement, we developed a new computational method for genetic analysis in mice. This method identifies the genetic basis for phenotypic differences among inbred mouse strains in a single day $[9,18]$. This method can be utilized for pharmacogenomic studies in mice by measuring a pharmacokinetic, pharmacodynamic or toxic response to a drug across a panel of inbred mouse strains. Genetic factors are then computationally predicted by identifying genomic region where the pattern of genetic variation correlates with the distribution of trait values among the inbred strains analyzed [18]. This method rapidly identified the genetic basis for a variety of phenotypic traits that varied among inbred mouse strains, including a polymorphism that regulated the pharmacodynamic response to aromatic hydrocarbon exposure [9]. We are now extending this to analyze the pharmacokinetic profile of a test drug across 13 inbred mouse strains. This computational method was unable to analyze the rate of parent compound disappearance across the inbred strains. At least 9 different intermediate metabolites were produced, and many different genetic factors contributed to the metabolism of the test drug. However, computational analysis of the concentration of selected intermediate metabolites enabled identification of a genetic factor regulating the metabolism of this test drug (unpublished data). The formation of each intermediate metabolite was controlled by a very limited set of genetic variables. Based upon these early results, we believe that analysis of the intermediate metabolites formed after administration of a drug to a panel of inbred strains can enable identification of metabolic factors regulating the metabolism of many medications. Characterization of a pharmacodynamic or toxic response to a medication among the inbred strains will enable this computational genetic method to identify genetic factors regulating the response to a medication. However, there are significant interspecies differences in drug metabolism that may limit our ability to translate metabolic factors identified in mouse to man. Because of this, it is likely that murine genetic variants that affect drug response that are identified by this computational method, rather than those affecting drug metabolism, will be of greater value over the long term.

\section{Expert opinion}

We used a quantitative model to demonstrate the advantages of using a genetically-guided dose adjustment strategy in clinical trials. Utilization of pharmacogenetic data resulted in increased efficacy and decreased toxicity in the treated population. To enable pharmacogenomic data to be more widely utilized in clinical practice, the genetic variables affecting the mechanism of action, disposition or toxicity of commonly used medications must be identified. A recently described murine haplotype-based computational genetic analysis method has been utilized to facilitate the identification of these genetic factors. As this tool is improved and becomes widely utilized for pharmacogenomic analysis, the rate of discovery of genetic factors regulating the metabolism and response to commonly used prescription medications will increase.

\section{Outlook}

With a simulation of a clinical trial, we demonstrate that pharmacogenomic information can optimize drug and dosage selection for individuals within a treated population. Although not 
widely utilized in areas of clinical practice at present, there are already examples where pharmacogenomic data has been used to optimize drug selection. There is a potential speedbump on the road toward increased utilization of pharmacogenomic information in clinical practice. The genetic factors regulating the response to many commonly used medications have yet to be discovered and comparative genomics may contribute much to this field. Application of current technology and capabilities should enable significant movement along the road toward optimal utilization of pharmacogenomic knowledge over the next three to five years.

\section{Highlights}

- A quantitative simulation demonstrated that utilization of pharmacogenomic information improves clinical trial design and treatment outcome. Incorporation of a genetically-guided dose adjustment strategy into a clinical trial significantly increased treatment efficacy and reduced toxicity.

- Genetic factors regulating the disposition, mechanism of action, and toxicity for many commonly used medications can be identified by several methods, including mouse genetics. A murine haplotype-based computational genetic analysis method may accelerate the rate of discovery of these clinically important pharmacogenomic factors.

- Currently available technologies should enable the discovery of genetic factors regulating the response to many commonly used drugs over the next 3 to 5 years. These discoveries should be translated into pharmacogenomic tests that aid in optimization of medication and dosage selection.

\section{Text BOX I}

\section{The simulations for the Phase II trial were based upon the following formula:}

- $\mathrm{Cp}_{\mathrm{i}}$, the plasma drug concentration in the ith individual following a dose of 1 unit: Let $\mathrm{r}=$ random number $(0,1)$

$$
C p_{i}=\left(\begin{array}{rr}
1.0 & \text { if }(r<0.64) \\
1.5 & \text { if }(r \geq 0.64 \text { and } r<0.96) \\
2.0 & \text { if }(r \geq 0.96)
\end{array}\right) \times e^{\varepsilon}
$$

where $\varepsilon \sim \mathrm{N}(0,0.04)$ where $0.64,0.32$, and 0.04 represent the frequency of the $(+/+),(+/-)$ and $(-/-)$ individuals in the population, respectively.

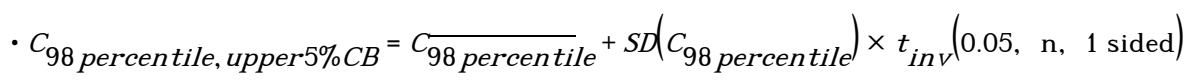

$C_{98 \text { percentile }}$, the 98 percentile concentration or the upper $2 \%$ concentration in each simulation; $C_{98}$ percentile,upper $5 \% \mathrm{CB}$, the top $5 \%$ confidence bound of $C_{98}$ percentile across the 250 simulations; $\mathrm{t}_{\mathrm{inv}}$ : the inverse of the one tailed, $5 \%$ Student $\mathrm{t}$ distribution, for $\mathrm{n}$ degrees of freedom;n, degrees of freedom, or the number of individuals in each simulated trial.

- Therefore, the dose in the genetically uninformed phase II trial was:

$$
\text { Dose }=\frac{12}{C_{98 \text { percentile, upper } 5 \% C B}}
$$

The same analyses were performed in the genetically informed trials, except

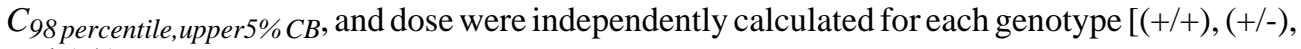
and $(-/-)]$. 


\section{TEXT BOX II}

\section{The simulations for the Phase III trial were based upon the following formula:}

- The plasma drug concentrations observed with each dose were determined for the genetically uninformed trial as: Let $r=$ random number $(0,1)$

$$
C p_{i}=\left(\begin{array}{rr}
1.0 & \text { if }(r<0.64) \\
1.5 & \text { if }(r \geq 0.64 \text { and } r<0.96) \\
2.0 & \text { if }(r \geq 0.96)
\end{array}\right) \times \text { Dose } \times e^{\mathcal{E}}
$$

where $\varepsilon \sim \mathrm{N}(0,0.04)$

- The same analyses were performed in the genetically informed trials, except the dose were independently adjusted for each genotype $[(+/+),(+/-)$, and $(-/-)]$.

- The fraction of patients in which the drug was efficacious or toxic was determined by plasma drug concentration. The drug was subtherapeutic $(<7)$, therapeutic (7-12), or toxic $(>12)$.

\section{Acknowledgement}

Y.G. was supported by a grant (1 R01 GM06885-01A1) from the NIGMS awarded to G.P.

\section{References}

1. Baetz K, McHardy L, Gable K, et al. Yeast genome-wide drug-induced haploinsufficiency screen to determine drug mode of action. Proc. Natl. Acad. Sci. U. S. A 2004;101:4525-4530. [PubMed: 15070751]

2. Brenner C. Chemical genomics in yeast. Genome Biol 2004;5:240. [PubMed: 15345040]

3. Eisenhauer EA. From the molecule to the clinic--inhibiting HER2 to treat breast cancer. N. Engl. J. Med 2001;344:841-842. [PubMed: 11248162]

4. Hetherington S, McGuirk S, Powell G, et al. Hypersensitivity reactions during therapy with the nucleoside reverse transcriptase inhibitor abacavir. Clin. Ther 2001;23:1603-1614. [PubMed: 11726000]

5. Ingelman-Sundberg M. Genetic polymorphisms of cytochrome P450 2D6 (CYP2D6): clinical consequences, evolutionary aspects and functional diversity. Pharmacogenomics J 2005;5:6-13. [PubMed: 15492763]

6. Karp CL, Grupe A, Schadt E, et al. Identification of complement factor 5 as a susceptibility locus for experimental allergic asthma. Nat. Immunol 2000;1:221-226. [PubMed: 10973279]

7. Klein RF, Allard J, Avnur Z, et al. Regulation of bone mass in mice by the lipoxygenase gene Alox 15 . Science 2004;303:229-232. [PubMed: 14716014]

8. Klotz U, Schwab M, Treiber G. CYP2C19 polymorphism and proton pump inhibitors. Basic. Clin. Pharmacol. Toxicol 2004;95:2-8. [PubMed: 15245569]

9. Liao G, Wang J, Guo J, et al. In silico genetics: identification of a functional element regulating H2Ealpha gene expression. Science 2004;306:690-695. [PubMed: 15499019]

10. Lum PY, Armour CD, Stepaniants SB, et al. Discovering modes of action for therapeutic compounds using a genome-wide screen of yeast heterozygotes. Cell 2004;116:121-137. [PubMed: 14718172]

11. McKinnon EJ, James IR, John M, Mallal SA. Viral load detectability profiles for HIV infection. Stat. Med 2003;22:385-396. [PubMed: 12529870]

12. Murphy MP, Beaman ME, Clark LS, et al. Prospective CYP2D6 genotyping as an exclusion criterion for enrollment of a phase III clinical trial. Pharmacogenetics 2000;10:583-590. [PubMed: 11037800]

13. Patarca R, Isava A, Campo R, et al. Human immunodeficiency virus type 1 pharmacogenomics in clinical practice: relevance of HIV-1 drug resistance testing (Part 1). J. Environ. Pathol. Toxicol. Oncol 2003;22:201-234. [PubMed: 14529094]

14. Payne DA, Bryant BJ. HIV pharmacogenomics: closer to personalized therapy? Am J Pharmacogenomics 2004;4:141-150. [PubMed: 15174895] 
15. Peltz G, Usuka J. From mouse genetics to human therapeutics. Curr. Opi. in Drug Disc. \& Dev 2005;8

16. Rozzo SJ, Allard JD, Choubey D, et al. Evidence for an interferon-inducible gene, Ifi202, in the susceptibility to systemic lupus. Immunity 2001;15:435-443. [PubMed: 11567633]

17. Slamon DJ, Leyland-Jones B, Shak S, et al. Use of chemotherapy plus a monoclonal antibody against HER2 for metastatic breast cancer that overexpresses HER2. N. Engl. J. Med 2001;344:783-792. [PubMed: 11248153]

18. Wang, J.; Peltz, G. Haplotype-Based Computational Genetic Analysis in Mice. In: Peltz, G., editor. Computational Genetics and Genomics: New Tools for Understanding Disease. Humana Press Inc; Totowa, NJ: 2005. p. 51-70.

19. Watters JW, McLeod HL. Murine pharmacogenomics: using the mouse to understand the genetics of drug therapy. Pharmacogenomics 2002;3:781-790. [PubMed: 12437480]

20. Wilson SG, Bryant CD, Lariviere WR, et al. The heritability of antinociception II: pharmacogenetic mediation of three over-the-counter analgesics in mice. J. Pharmacol. Exp. Ther 2003;305:755-764. [PubMed: 12606637] 


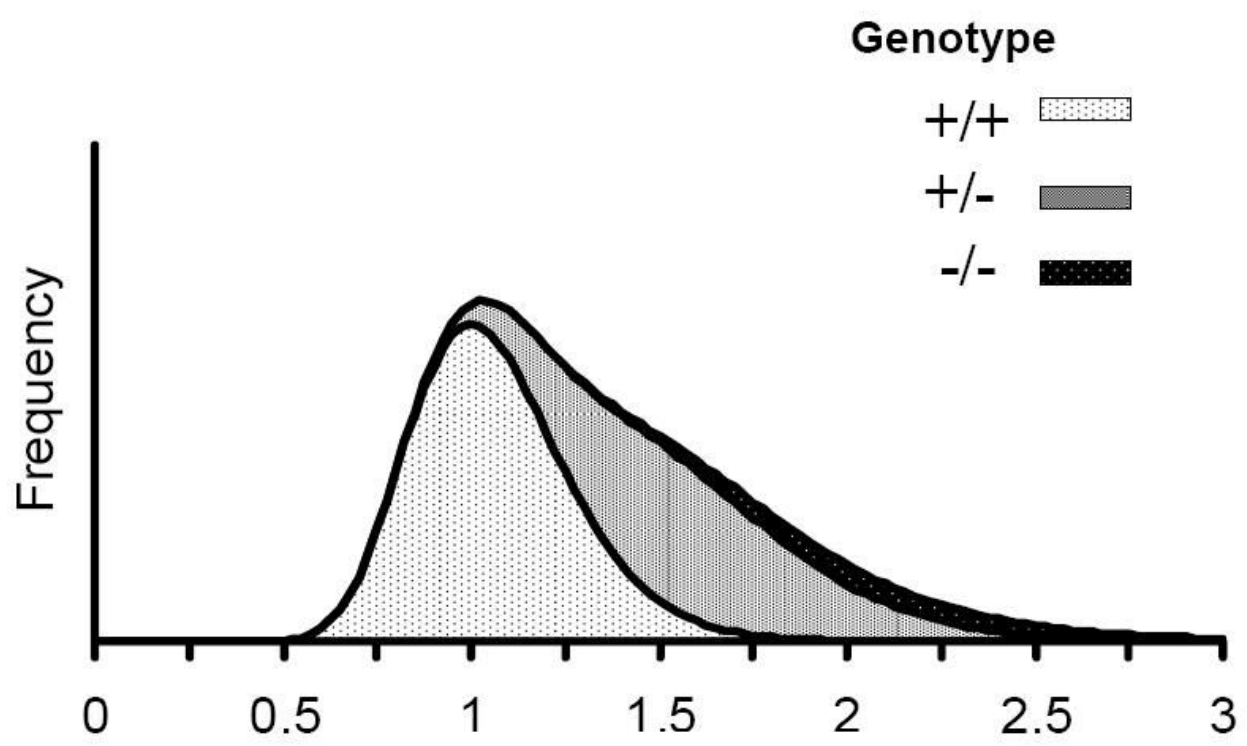

\section{Plasma drug concentration}

Figure 1.

Overall distribution of drug response in a population with different genotypes. A hypothetical drug is administered in a 1 unit bolus dose to a population of individuals that have homozygous wildtype, heterozygotes and homozygous recessive alleles of a drug-metabolizing gene. The wild type allele, has an allele frequency of 0.8 , is dominant, and results in a rapid rate of metabolism of this drug. A log normal distribution with a $20 \% \mathrm{CV}$ in the individual variations of plasma drug concentration is assumed. The influence of each genotype on the overall distribution of drug response is weighted by the corresponding frequency of each genotype. 


\section{Phase III Trial Simulations}

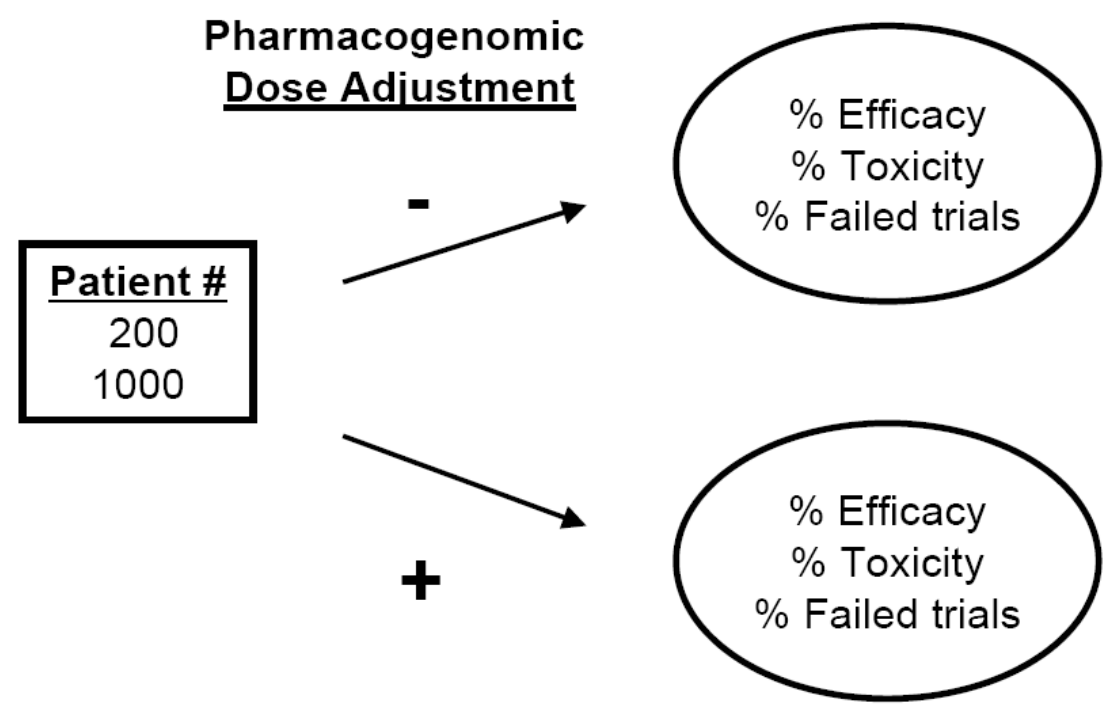

Figure 2.

Diagrammatic overview of the clinical trial simulations that were quantitatively modeled. (A) In phase II simulations, a 1 unit dose of drug is administered to the indicated number of patients. The plasma drug concentration is then measured and used to calculate the dose for maximum efficacy for all treated patients (without genotype data), or the dose for maximum efficacy for each of the three different genotypes within the treated population (with genotype data). (B) Using the doses established in the phase II trial, the subsequent pivotal phase III trials perform simulations with 200 or 1000 subjects to assess the efficacy and safety of the drug. 


\section{Genetically Uninformed Design}

\section{Table I}

\begin{tabular}{|c|c|c|c|c|c|c|c|}
\hline \multirow{2}{*}{$\begin{array}{c}\text { Subjects } \\
\text { in Phase } \\
\text { II }\end{array}$} & \multirow{2}{*}{$\begin{array}{c}\text { Dose } \\
\text { calculated } \\
\text { from Phase II } \\
\text { simulations }\end{array}$} & \multicolumn{3}{|c|}{ Phase III: 200 Subjects } & \multicolumn{3}{|c|}{ Phase III 1000 Subjects } \\
\hline & & Efficacy & Toxicity & Failed Trials & Efficacy & Toxicity & Failed Trials \\
\hline 10 & 4.86 & $24 \%$ & $0.6 \%$ & $2 \%$ & $24 \%$ & $0.80 \%$ & $0 \%$ \\
\hline 100 & 4.94 & $25 \%$ & $0.8 \%$ & $4 \%$ & $25 \%$ & $0.90 \%$ & $0 \%$ \\
\hline 200 & 5.01 & $27 \%$ & $0.9 \%$ & $6 \%$ & $26 \%$ & $1.00 \%$ & $0 \%$ \\
\hline 1000 & 5.17 & $29 \%$ & $12.0 \%$ & $17 \%$ & $29 \%$ & $1.40 \%$ & $5 \%$ \\
\hline
\end{tabular}




\section{Table II}

Genetically Informed Design

\begin{tabular}{|c|c|c|c|c|c|c|c|c|c|}
\hline \multirow{2}{*}{$\begin{array}{c}\text { Subjects } \\
\text { in Phase } \\
\text { II }\end{array}$} & \multicolumn{3}{|c|}{ Dose calculated from Phase II simulations } & \multicolumn{3}{|c|}{ Phase III: 200 Subjects } & \multicolumn{3}{|c|}{ Phase III 1000 Subjects } \\
\hline & WT & Heterozygous & Homozygous & Efficacy & Toxicity & Failed Trials & Efficacy & Toxicity & Failed Trials \\
\hline 100 & 7.40 & 4.94 & N/A & & & & & & \\
\hline 200 & 7.45 & 4.95 & 3.82 & $62 \%$ & $0.7 \%$ & $3 \%$ & $61 \%$ & $0.90 \%$ & $0 \%$ \\
\hline 1000 & 7.72 & 5.06 & 3.75 & $66 \%$ & $1 \%$ & $11 \%$ & $66 \%$ & $1.30 \%$ & $2 \%$ \\
\hline
\end{tabular}

